\title{
¿Qué nos han enseñado los estudios de seguimiento de toxicómanos?
}

\author{
Marina, P. \\ Médico de los servicios de salud mental del Principado de Asturias. Unidad de tratamiento de toxicomanías de Oviedo. \\ Enviar correspondencia a: \\ Pedro Marina. C/ Unamuno 19, $8^{\circ}$ A. 33010 Oviedo.
}

\begin{abstract}
Resumen
A finales de la década de los ochenta y en los primeros años de los noventa se llevaron a cabo en España los primeros estudios de seguimiento de toxicómanos con el ánimo de conocer el perfil de los pacientes y la eficacia de los tratamientos. Sus resultados aportaron información de primera mano acerca del colectivo de drogodependientes, sus problemas y la respuesta asistencial. En este artículo se revisa y comenta la influencia que estos estudios ejercieron en la organización de los recursos asistenciales y en la adecuación de la oferta terapéutica a las necesidades de los pacientes y se señalan los objetivos de futuros estudios de seguimiento a la luz de la experiencia adquirida y las circunstancias actuales.
\end{abstract}

Palabras clave: Drogodependiente. Seguimiento. Evaluación de tratamientos. Demanda asistencial. Oferta terapéutica.

\begin{abstract}
At the end of the eighties and in the early nineties they were carried out the first follow-up of heroin addicts in Spain. The studies goals were to characterize the patients and to evaluate the treatments efficacy. The results of this studies gave information about the population of heroin addicts and the therapeutic organization in Spain. In this paper we review and we comment on the influence of follow-up studies results over the clinical responses and the therapeutic offer adaptation to the patients problems. We point out objectives news follow-up studies from previous experience.
\end{abstract}

Key words: Drug addict. Follow-up. Evaluation of treatments. Assistance demand. Therapeutic programs.

\section{INTRODUCCIÓN}

E n la segunda mitad de los años ochenta y en la primera de los noventa se llevaron a cabo en España un número importante de estudios de seguimiento de drogodependientes $(1,2,3,4,5,6,7$, 8) cuyos resultados aportaron un notable conocimiento acerca de las personas con problemas de drogas en nuestro medio: características, dificultades asociadas, respuesta a los programas de tratamiento y evolución; a la par que introducían las bases metodológicas para la realización de estudios similares. Existían experiencia y estudios de este tipo en otros países $(9,10,11$, $12,13)$ pero no en el nuestro, donde el fenómeno de las drogas había hecho eclosión a finales de la década de los setenta.

Conviene recordar que estudio de seguimiento es toda investigación en la que una muestra es identificada en el tiempo y posteriormente localizada en persona o por registros para saber qué ha ocurrido en el intervalo (14) y que tiene como objetivos todos o algunos de los siguientes:

a) Conocer las características de los pacientes que solicitan tratamiento, detectar diferencias y definir tipologías.

b) Obtener información sistematizada sobre la situación de los pacientes después de dejar un programa de tratamiento.

c) Describir los servicios de tratamiento en un programa.

d) Conocer la opinión de los pacientes sobre los programas de tratamiento.

e) Evaluar los efectos del tratamiento.

f) Comparar distintas modalidades de tratamiento.

Pero, ¿cuál fue el impacto de estos estudios?, ¿qué influencia ejercieron sus resultados en los profesionales y los recursos de atención y tratamiento?, y a partir de la experiencia y circunstancias actuales, ¿hacía qué objetivos deben orientarse futuros estudios? Para responder a estas preguntas se revisan y 
comentan los estudios de seguimiento más relevantes, la evolución del perfil y las necesidades asistenciales de los drogodependientes y las características de los recursos de tratamiento.

\section{DEMANDA Y OFERTA ASISTENCIAL}

Sabemos que la intervención en drogodependencias es en ocasiones una intervención improvisada, fruto de decisiones políticas, de la presión social o de quien asegura tener la receta para resolver el problema. No es menos cierto, que los profesionales de las drogas son con frecuencia excesivamente conservadores y resistentes a modificar el modus operandi. Sin embargo, existe un conjunto de conocimientos extenso y ordenado fruto de la investigación y la práctica que debe servir de base a toda actuación.

Los primeros estudios de seguimiento, fueron estudios de verificación porque pretendían confirmar estadísticamente los perfiles de los toxicómanos españoles observados en la clínica y comprobar la influencia en los mismos de los tratamientos. Con ellos se pusieron de manifiesto las características sociodemográficas (edad, sexo, convivencia, estudios, profesión, empleo, etc.) y del consumo de drogas de los drogodependientes (sustancias usadas, frecuencia de consumo, vias de administración, etc.), los problemas legales (actividad delictiva, detenciones y encarcelaciones) y de salud relacionados con el consumo (prevalencia de sida, hepatitis, tuberculosis, algunas enfermedades mentales, etc.), la mortalidad, la proporción de sujetos que alcanzaba la abstinencia y las variables con ella relacionadas. Así, se constató que la dependencia es un itinerario que discurre por periodos de consumo y abstinencia, de modo que el porcentaje de abstinentes en un momento determinado podía oscilar entre el $40 \%$ y el $50 \%$, que los tratamientos ofrecían buenos resultados y que con la abstinencia mejoraba la salud, la situación socio-familiar y laboral y se reducían los problemas legales $(2,3,4,5,6,8)$.

Pero además, estos estudios de seguimiento proporcionaron información sobre otros aspectos muy importantes, como el nivel de adecuación de la oferta de tratamiento a los problemas y demandas de los pacientes. A finales de los años ochenta se estaba desarrollando y completando la red de atención al drogodependiente y hasta ella llegaban personas con una larga historia de uso de drogas (en muchos casos iniciada a finales de los setenta), de modo que la media de años trascurridos desde el inicio del consumo hasta el primer tratamiento superaba los cinco años. Tenían, pues, una escasa experiencia terapéutica y además marcados signos de desarraigo: bajísimo nivel de instrucción (35\% con estudios primarios), pobre formación profesional y escasa experiencia laboral y una dilatada carrera delictiva (alrededor del 60\% con antecedentes de ingresos en prisión) (1, 2, 3, 4, 8). La mayoría de los programas de tratamiento trabajaban con objetivos de abstinencia y cualquiera que quisiera seguirlos debía reunir los requisitos exigidos y cumplir con sus actividades. De un lado estaba el perfil de los pacientes, de otro los procesos de los programas y la aproximación entre uno y otro se producía por la adaptación del primero al segundo, si podía, y excepcionalmente al contrario.

Los resultados de los pocos trabajos que, como el realizado en Asturias, estudiaron algún indicador de adecuación entre las necesidades de la demanda y la respuesta terapéutica, demostraron que, en efecto, un número importante de toxicómanos no conseguía adaptarse a las exigencias de los tratamientos y rompían el contacto: el $54 \%$ de las personas estudiadas en el citado trabajo no llegaron a iniciar el tratamiento solicitado en los servicios de salud mental asturianos (15). Pero por entonces, había escaso interés por estas cuestiones y los estudios de seguimiento pasaban de puntillas sobre ellas. El centro de interés estaba en estudiar lo que sucedía a partir de iniciar un tratamiento y se dejaba de lado todo lo que acontecía previamente. La "falta de motivación" del paciente o el "que no hubiera tocado fondo", eran los argumentos más generalizados y habituales para justificar los abandonos. Sin embargo, al comparar las características de las personas que iniciaban y de las que no iniciaban un tratamiento, se observaba que entre las segundas había personas con indicadores de más "gravedad" (más jóvenes, de incio más precoz en el uso de drogas y peor situación profesional y laboral)y, por tanto, candidatas a beneficiarse más del tratamiento $(15,16)$.

En definitiva, lo que estos estudios de seguimiento reflejan es que el objetivo principal del trabajo terapéutico era la abstinencia, el medio, el programa de tratamiento y el paciente alguien a encajar en éste para llegar a aquel. Por tanto, la demanda debía adecuarse a la oferta y no a la inversa. Se presta poco interés a la comorbilidad psiquiátrica y a otras circunstancias personales asociadas al mantenimiento del consumo y las recaídas y se buscan variables de buen pronóstico a partir de los sujetos abstinentes.

\section{UN NUEVO PARADIGMA ASISTENCIAL}

Durante este tiempo un importante número de drogodependientes fue acumulando experiencias de tratamiento (17), fracasos y de esta manera desmoralización y deterioro personal y socio-familiar (el $60 \%$ de la población entrevistada en Asturias había hecho más de dos tratamientos entre 1987 y 1990 y el 20\% más de cuatro) ${ }^{15}$, con una altísima tasa de infección por el virus del sida (mayor del 40\% en la mayoría de los estudios (18) y una elevada frecuencia de problemas legales. Además, cuando se utilizaban procedimientos de screening para la detección de casos psiquiátricos las cifras de probables casos entre los drogodependientes supera- 
ban con mucho a los encontrados en estudios de población general (64\% frente al 35\%) (15).

Si bien estas evidencias reclamaban otros modelos de atención, fue principalmente la difusión del sida y la infección del VIH entre el colectivo de toxicómanos el motor del cambio. Con el cambio se produjo algo más que una modificación en las técnicas de tratamiento, supuso un trascendente giro de mentalidad al aceptarse de manera generalizada que el tratamiento de los adictos podía tener como objetivo la reducción de los daños derivados del consumo y no sólo la abstinencia. De este modo el foco de las intervenciones terapéuticas se desplazó de la abstinencia al sujeto, descubriendo a una persona con problemas y una vivencia de los mismos que requería ser escuchada.

Paralelamente, la investigación de la comorbilidad psiquiátrica en usuarios de drogas, desvelaba la importancia de los trastornos duales en el origen y mantenimiento de la conducta adictiva (19). Asimismo, el estudio de los procesos individuales de cambio para el abandono del consumo de drogas, identificaba distintas etapas, desde la precontemplación a la actuación y el mantenimiento, que implican actitudes diferentes del consumidor y que requieren intervenciones distintas del terapeuta $(20,21)$. En suma, se dieron pasos fundamentales para contemplar al consumidor de drogas como un sujeto afecto por un trastorno íntimamente relacionado con la salud psíquica que pasa por momentos distintos en relación a su capacidad y deseo de cambio.

En consecuencia, la exploración psicopatológica y la identificación de la situación del paciente en el ciclo de cambio se convierten en dos variables esenciales para la indicación terapéutica. Esto implica avanzar hacia la profesionalización de la intervención en drogodependencias, rechazar el "todo vale para todos" y reconocer que el fracaso terapéutico no es responsabilidad exclusiva del paciente. Salvo que se siga considerando al toxicómano como un vicioso o un "enfermo moral" es díficil aceptar que el tratamiento pasa por la catarsis purificadora de programas basados en la disciplina moral. Es cierto que cualquier centro de tratamiento tiene su porcentaje de "curaciones", pero también algunos enfermos mentales graves mejoraban cuando eran recluidos en manicomios o antes en hospitales de piedad. No se trata sólo de resultados sino de reconocer el derecho del paciente a recibir un tratamiento digno y acorde a lo que el conocimiento científico ha permitido conocer de su enfermedad.

\section{HACIA LA ARMONIZACIÓN ENTRE DEMANDA ASISTENCIALY OFERTA TERAPÉUTICA}

En lo que se refiere a los hábitos de consumo, a lo largo de los años noventa se configuran nuevas modalidades de uso de drogas legales e ilegales que resul- tan claramente diferentes de las mantenidas en la década anterior (22). Como consecuencia, a los problemas relacionados con el consumo de heroína, acentuados por las graves dificultades sociosanitarias de quienes mantienen el consumo después de muchos años (sida, desarraigo social, etc.), se añaden otros de más reciente cuño como las derivadas de los efectos inmediatos y a largo plazo del alcohol y de otras drogas, especialmente las estimulantes, en un colectivo de jóvenes que las usan en el contexto de las relaciones de grupo y en los momentos de ocio y tiempo libre. Estas drogas tienen un especial efecto desestructurador en sujetos con alguna fragilidad psíquica y socio-familiar, de modo que pueden desencadenar graves alteraciones personales y en el funcionamiento social con el consecuente riesgo de cronificación.

De este modo, conviven dos perfiles marcadamente diferentes de usuarios de drogas con problemas y demandas de tratamiento muy distintas. Por un lado el heroinómano crónico, con una larga historia de consumo, múltiples tratamientos y numerosos problemas legales, multidemandante, que hace uso de múltiples recursos sociales y sanitarios, en especial los de reducción del daño (metadona, intercambio de jeringuillas, pisos de acogida, etc.) y los destinados a poblaciones marginales (albergues, cocina económica, etc.). De otro lado, jóvenes consumidores de alcohol u otras drogas, cuya frecuencia de uso va desde del abuso al exceso, que demandan tratamiento porque presentan síntomas de síndrome de dependencia o de algún trastorno mental o del comportamiento producido o agravado por las drogas. Son sujetos que acuden a las redes normalizadas de tratamiento y no identifican sus dificultades con las de los heroinómanos. Su vinculación a los centros públicos de tratamiento es elástica (aparecen y desaparecen), díficil de incardinar al sistema tradicional de atención ambulatoria. Por eso, se necesita pensar en nuevas modalidades de atención para estos jóvenes, para atenuar el riesgo que corren de deterioro y cronificación (23). Pero además, a estos distintos perfiles se deben añadir las diferencias de género. La investigación y la experiencia clínica están demostrando que los hombres y las mujeres tienen tasas de inicio en el consumo de drogas diferentes, distintos patrones de uso de drogas, diferencias en las consecuencias del uso crónico de sustancias, e incluso estilos distintos de abandono del uso de drogas (24).

En esta realidad compleja de los nuevos patrones de consumo, de distintos perfiles de consumidores, de estrategias terapéuticas innovadoras, es donde deben enmarcarse los nuevos estudios de seguimiento. Su realización debe ofrecer una visión de conjunto, del resultado de la interacción entre drogodependientes y programas de tratamiento para avanzar en la necesaria armonización entre las respuestas terapéuticas y las demandas asistenciales, así como informa- 
ción para contrastar la eficacia de los distintos estilos de tratamiento. Con esta finalidad los futuros estudios de seguimiento deberían diseñarse para evaluar periódicamente la aplicación y resultados de los programas de tratamiento, de manera similar a como se hace en otros países, en especial los EE.UU., donde periódicamente se llevan a cabo macro-estudios de evaluación de tratamientos. Hasta el momento han realizado tres, el Drug Abuse Reporting Program (DARP) (11), el Treatment Outcome Prospective Syudy (TOPS) (13), y más recientemente el Drug Abuse Treatment Outcome Study (DATOS) (26).

En estos estudios de seguimiento de nuevo cuño se debería:

1) Prestar atención a la presencia de patología psiquiátrica en los drogodependientes.

2) Estudiar la influencia de los trastornos psíquicos en la pronóstico y la evolución de la conducta adictiva.

3) Estudiar la evolución de los trastornos mentales con la presencia y en la ausencia del consumo de drogas.

4) Analizar los procesos de indicación terapéutica.

5) Investigar el efecto de un proceso de tratamiento indicado teniendo en cuenta el sexo, la comorbilidad psiquiátrica y la etapa del sujeto en el ciclo de cambio.

4) Medir la eficacia del tratamiento no sólo en abstinencia, sino también con parámetros como la retención y la satisfacción de los pacientes.

\section{BIBLIOGRAFÍA}

(1) MELGAREJO,M.; SANAHUJA,P.; MASFERRER,J. y col. Estudio de seguimiento en población heroinómana. Fons Informatiu, 1986,1, pp.1-38.

(2) GUARDIA,J.; MASIP,J.; VILADRICH,C. y col. Estudio de seguimiento de adictos a la heroína a los tres años de su admisión a tratamiento. Comunidad y Drogas, 1987,3, pp.61-79.

(3) SANCHEZ-CARBONELL, J., CAMI, J. \& BRIGOS, B. Follow-up of heroin addicts in Spain (EMETYST Project): results 1 year after teatment admission, British Journal of Addiction, 1988, 83.

(4) MARINA,P.; VAZQUEZ-BARQUERO, J.L.; JIMENEZ,J. y col. Los beneficios de la abstinencia: un estudio de seguimiento de adictos a la heroína. Adicciones, 1996, vol 8, n³, pp.295-309.

(5) GRUPO INTERDISCIPLINAR SOBRE DROGAS. Los estudios de seguimiento: una experiencia metodológica. Madrid. Editorial Fundamentos. 1992. 351 pp.

(6) TORRENS,M. y col. Estudio de seguimiento de pacientes dados de alta en una UDH, Cuadernos del Centro
Valenciano de Documentación sobre Drogodependencias, 1993, Marzo, pp. 147-148.

(7) SAN, L. y col., Follow-up after a six-month maintenance period on naltrexone versus placebo in heroin addicts, British Journal of Addiction, 1991, 86, 8, pp. 983-990.

(8) GARCIA,E.; ROLDAN,G.; COMAS,D., Un estudio de seguimiento de la Comunidad terapéutica HaizeGain, 1982-1992, San Sebastián, Comunidad Terapéutica Haize-Gain, 1995, 99 pp.

(9) VAILLANT, G.E. A twenty-year follow-up of New York narcotic addicts, Archives of General Psychiatry, 1973, 29, pp. 237-241.

(10) STIMSON, G., OPPENHEIMER, E. \& THORLEY, A. Seven year follow-up of heroin addicts: drug use and outcome, British Medical Journal, 1978, 1, pp. 11901192.

(11) SIMPSON, D. National treatment system evaluation based on the drug abuser reporting program (DARP) follow-up research, in: F.J. TIMS \& J.P. LUDFORD (Eds) Drug Abuse Treatment Evaluation: Strategies, Progress and Prospects, 1984, pp. 29-41, Washington, DC, National Institute of Drug Abuse.

(12) HAASTRUP, S. \& JEPSEN, P. Eleven year follow-up of 300 young opioid addicts, Acta Psychiatrica Scandinavica, 1988, 77/1, pp. 22-26.

(13) HUBBARD, R., RACHAL, J., CRADDOCK, S. \& CAVANAUGH, B. Treatment outcome prospective study (TOPS): client characteristics and behaviors before, during, and after treatment, in: F.J. TIMS \& J.P. LUDFORD (Eds) Drug Abuse Treatment Evaluation: Strategies, Progress and Prospects, 1984, pp. 42-68, Washington, DC, National Institute of Drug Abuse.

(14) JOHNSTON, L., NURCO, D. \& ROBINS, L.N. Conducting follow-up research on drug treatment programs, Washington DC, National Institute of Drug Abuse, 1977, 191 pp.

(15) MARINA, P. Adictos a la heroína en Asturias, Oviedo, Servicio de Publicaciones del Principado de Asturias, 1993, $133 \mathrm{pp}$.

(16) APODACA,I., y col., Características de los toxicómanos que no inician o interrumpen tempranamente el tratamiento, Adicciones, 1995, 7, 2, pp. 159-167.

(17) IRAURGI,I. Cambios en las pautas de consumo de drogas en usuarios de heroína atendidos en un servicio ambulatorio de Bilbao (España). Evolución durante el periodo 1979-1995. Revista Española de Drogodependencias, 1997, vol.22, 1, pp.13-23.

(18) DELEGACIÓN DEL GOBIERNO PARA EL PLAN NACIONAL SOBRE DROGAS. Infección por VIH en usuarios de drogas. Madrid. Ministerio de Sanidad y Consumo. Publicaciones, Documentación y Biblioteca. 1991. 194 $\mathrm{pp}$.

(19) ROUNSAVILLE, B.J., WEISSMANN, M.M., KLEBER, H.D., WILBER, C. Heterogeneity of psychiatric diagnosis in treated opiate addicts, Archives of General Psychiatry, 1982, 39, pp. 161-166.

(20) PROCHASKA, J.O.; DiCLEMENTE,C. Transtheoretical therapy: Toward a more integrative model of change. 
Psychoterapy: theory, Research and Practice, 1982, 19, pp.276-278.

(21) TRUJOLS,J.; TEJERO,A.; CASAS,M. Estructura factorial, consistencia interna y eficacia discriminativa del inventario de procesos de cambio para adictos a la heroína. Adicciones, 1997, vol.9, n³, pp.331-345.

(22) COMAS, D. Los jóvenes y el uso de drogas en la España de los años 90. Madrid. Ministerio de Asuntos Sociales. Instituto de la Juventud. 1994. 271 pp.

(23) ARNAU,C. y col., Políticas sobre drogas a partir de los resultados de un estudio longitudinal retrospectivo de adictos a la heroína (1982-1992). Adicciones, 1997, vol.8, n¹, pp.75-89.

(24) BARTHWELL, A.G. Treatment of Women, National Conference on Drug Addiction Treatment: From
Research to Practice (Abstracts), Maryland (USA), Abril 1998, Disponibles en: http://www.nida.nih.gov/ MeetSum/TX/TXinfo3.html\#Barthwell (citado 10 de enero de 1999)

(25) BERJANO,E., Diversos modelos de tratamiento para los diferentes tipos de adictos, Cuadernos del Centro Valenciano de Documentación sobre Drogodependencias, 1993, Marzo, pp. 161-164.

(26) MUELLER,M.D. and WYMAN,J.R., Study Sheds New Light on the State of Drug Abuse Treatment Nationwide. NIDA Notes (revista electrónica), 1997, vol.12, n5, Setiembre/Octubre, 20 pantallas. Disponible en http:// www.nida.nih.gov/NIDA_Notes/NNVol12N5/Study.html (citado 10 de enero de 1999). 
